\title{
Low Alpha Experiments at the ALS ${ }^{1}$
}

\author{
D. Robin*, R. Alvis*, and A. Jackson* \\ Lawrence Berkeley National Laboratory, Berkeley, CA 94720 USA \\ R. Holtzapple ${ }^{\dagger}$ and B. Podobedov ${ }^{\dagger}$ \\ Stanford Linear Accelerator Center, P.O. Box 4349, Stanford, CA, 94309, USA
}

\begin{abstract}
.
We present a modified, low alpha lattice for the Advanced Light Source where the quadrupole field strengths have been detuned to allow the momentum compaction factor to be varied smoothly from positive to negative values. With this low alpha lattice we decrease the momentum compaction factor by a factor of 5 to 0.0003 over normal operation resulting in a measured bunch length reduction of 2. We also measure the size the second order momentum compaction factor as well as store beam in a negative momentum compaction lattice. Streak camera measurements at positive and negative momentum compaction operation show longitudinal beam profile distributions that are in agreement with simulations by Fang et al (8).
\end{abstract}

\section{INTRODUCTION}

At the Advanced Light Source there is interest in decreasing the bunch length and increasing the peak current of electron bunches. Very short bunches (less than $1 \mathrm{ps)}$ promise coherent infrared radiation as well as better time resolution. High peak currents promise larger radiation power. Recent experimental work at UVSOR (1-3) and Super-ACO (3-5) have demonstrated that it is possible to decrease the bunch length in a storage ring by reducing the momentum compaction factor. In these experiments the momentum compaction factors were reduced by two orders of magnitude giving a reduction in bunch length of 10 . In addition, results at SuperACO have demonstrated that at negative values of the momentum compaction factor, there is less bunch lengthening than at positive values of the momentum compaction factor (3). Therefore it is possible to store larger peak currents at negative than at positive momentum compaction.

An experimental program to reduce the momentum compaction is underway at the Advanced Light Source (ALS). At this time we have succeeded in reducing the bunch length by a factor of two from that of normal operation. We have also stored beam in a negative momentum compaction configuration. In this paper we report on the current status of these experiments.

Presented at Micro Bunches: AWorkshop on the Production, Measurement and Applications of Short Bunches of Electrons and Positrons in Linacs and Storage Rings, Upton, L.I., New York, September 28-30, 1995.

\footnotetext{
${ }^{1}$ This work was supported by the Director, Office of Energy Research, Office of Basic Energy Sciences, Materials Sciences, Division of the U.S. Department of Energy under Contract Numbers DE-AC03-76SF00098* and DE-AC03-76SF00515 ${ }^{\dagger}$.
} 


\section{LATTICE}

The lattice of the ALS is built up of 12 triple bend achromat (TBA) cells. The layout of a typical cell can be seen in figure 1 . There is one bend family, B, Three quadrupole families, QF, QD, and QFA, and two sextupole families, SF and SD.

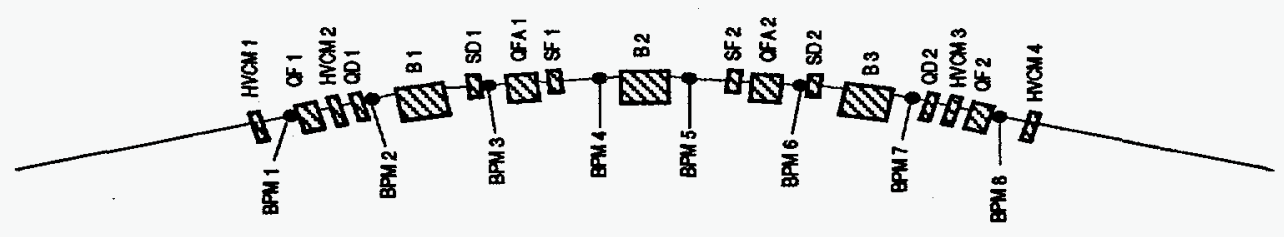

FIG. 1. Top-down view of a typical sector (1/12 of the ring).

The Twiss parameters for the lattice in its usual operation mode can be seen in figure 2 (a). For the usual operation mode the lattice is designed such that there is zero dispersion, $\eta$, in the straight sections to the outside of the outer bends and positive dispersion elsewhere.
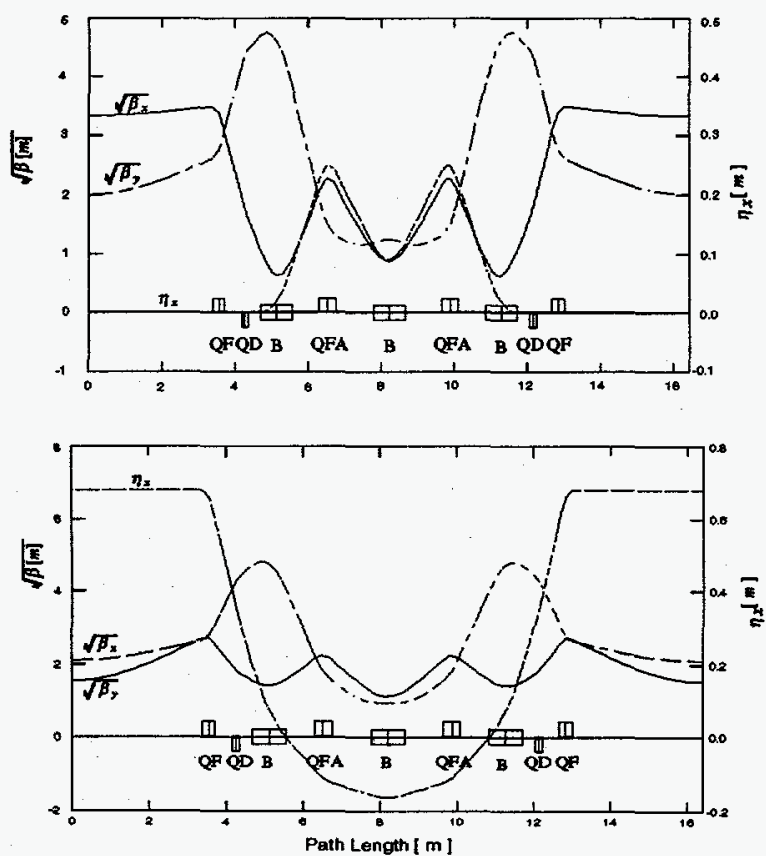

FIG. 2. Lattice functions for an ALS cell. a: normal ALS sector (top). b: modified sector (bottom).

Because the dispersion is positive in the bends, higher energy particles have a longer total orbital path length than lower energy particles. This is because higher energy particles travel radially outward of lower energy particles in the bends. The momentum compaction factor, $\alpha$, is a measure of the path length difference, $\Delta L$, of an off and on-energy particle relative to the energy difference, $\Delta E$, of an off and 


\section{DISCLAIMER}

Portions of this document may be illegible electronic image products. Images are produced from the best available original document. 
on-energy particle and is expressed as:

$$
\frac{\Delta L}{L_{o}}=\alpha \frac{\Delta E}{E_{o}}
$$

where $L_{o}$ and $E_{o}$ are the total path length and energy of the ideal particle respectively. To first order in $\Delta E / E_{o}$, the path length difference is given by the first order momentum compaction factor, $\alpha_{1}$ and is given as (6):

$$
\alpha_{1}=\int_{0}^{L_{o}} \frac{\eta}{\rho} d s,
$$

where $\rho$ is the radius of curvature of the bends and $\eta$ is the dispersion in the bends. Because the dispersion in the bends is positive (figure $2(\mathrm{a})$ ),$\alpha_{1}$ is positive.

For our experiment the initial aim was to find a new lattice configuration that $\alpha_{1}$ could be smoothly tuned from positive to negative values. We also imposed the constraint that we would only be allowed to vary the quadrupole field gradients $k_{Q F}, k_{Q D}$ and $k_{Q F A}$. In other words, we did not want to make physical changes to the lattice. With these aims and constraints we arrived at the lattice seen in figure 2 (b). In this modified or low alpha lattice the dispersion is positive in the straight sections and the outer bends and negative in the middle bend. So higher energy particles travel radially outward in the outside bends but radially inward in the middle bend. Therefore the higher energy particles "cut the corner" in the middle of the straight section and reduce their total path length. If the dispersion function is made more negative in the middle bend, the higher energy particles will "cut the corner" more, and the momentum compaction will be reduced further and can even become negative.

In table 1 a comparison is made between the parameters for normal operation lattice and the low alpha lattice. It is clear from table 1 that the properties of the lattices are very different. In particular for the low alpha lattice, the emittance is 10 times larger, and the horizontal tunes and chromaticities are lower.

In the low alpha lattice, the $\mathrm{QF}$ quadrupoles are located in a region of maximum dispersion, $\eta=0.7 \mathrm{~m}$ (figure $2(\mathrm{~b})$ ). Since the change in $\alpha_{1}$, resulting from a change in integrated quadrupole field $\Delta k_{l}$, is proportional to the square of the dispersion at the location of the quadrupole,

$$
\Delta \alpha_{1} \propto \Delta k_{l} \eta^{2},
$$

changing the QF's field is a very effective method of changing $\alpha_{1}$. In figure 3 we plot calculated $\alpha_{1}$ against $\mathrm{QF}$ excitation current, $\Delta I_{Q F}(7)$.

\begin{tabular}{||c|c|c|c||}
\hline Quantity & Symbol & Normal-Alpha Lattice & Low-Alpha Lattice \\
\hline Horizontal Tune & $\nu_{x}$ & 14.28 & 10.26 \\
Vertical Tune & $\nu_{y}$ & 8.18 & 8.15 \\
Natural Horizontal Chromaticity & $\nu_{x}^{\prime}$ & -25 & -11 \\
Natural Vertical Chromaticity & $\nu_{y}^{\prime}$ & -28 & -28 \\
Horizontal Emittance & $\epsilon_{x}$ & $4 \mathrm{~nm}-\mathrm{rad}$ & $40 \mathrm{~nm}-\mathrm{rad}$ \\
Dispersion at SF & $\eta_{S F}$ & $0.211 \mathrm{~m}$ & $-0.111 \mathrm{~m}$ \\
Dispersion at SD & $\eta_{S D}$ & 0.124 & -0.014 \\
Momentum Compaction & $\alpha_{1}$ & 0.0016 & 0.0003 \\
\hline \hline
\end{tabular}

TABLE 1. Parameters for the normal and low alpha lattices. 


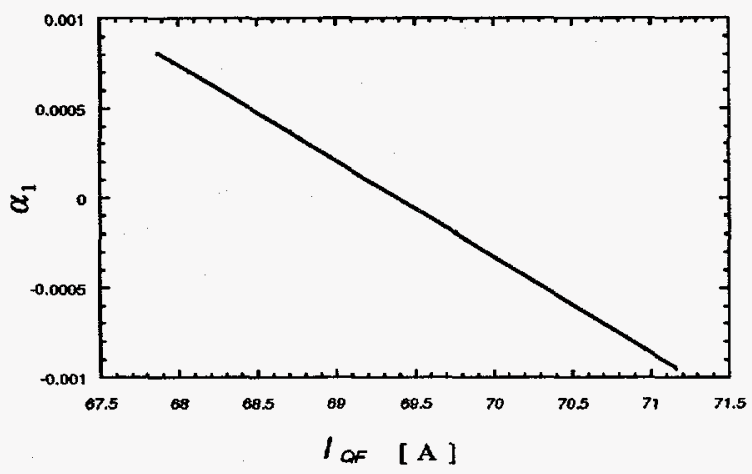

FIG. 3. Calculated value of $\alpha_{1}$ verses $Q F$ excitation current.

\section{EXPERIMENT}

In July and August of 1995 we performed four experiments where we studied the properties of this new lattice. In all of the experiments, the sextupoles were turned off. The first two experiments were dedicated to characterizing the low alpha lattice at larger values of $\alpha_{1}$. In the last two experiments we proceeded to reduce and even make $\alpha_{1}$ negative by adjusting the QF magnets.

\section{Characterizing the Low Alpha Lattice}

In the first experiment beam was successfully injected into the new lattice. Because of the large (negative) values of the chromaticity, it was very difficult to measure the tunes with a spectrum analyzer. Instead we used the 96 beam position monitors (BPMs) which are able to measure the beam orbit every turn. A bunch was then injected off-axis and its orbit location was recorded over a number of consective turns. From this data the integer and noninteger tunes were extracted. The tunes were measured to be $\nu_{x}=10.25$ and $\nu_{y}=8.45$. The resolution of the tune measurement was \pm 0.05 .

\section{Measurement of $\alpha_{1}$}

Synchrotron Tune. We were able to observe self-excited synchrotron frequency side bands about the revolution harmonic frequency. This was an indication of some instability in the beam. As a result we were able to measure the the synchrotron tune, $\nu_{s}$, without having to excite longitudinal oscillations.

The first order momentum compaction factor, $\alpha_{1}$, is proportional to the square of the synchrotron tune, $\nu_{s}^{2}$,

$$
\alpha_{1}=\frac{2 \pi E_{o}}{e h V_{\circ} \cos (\phi)} \nu_{s}^{2}
$$

where $V_{o}, h$ and $\phi_{o}$ are the peak voltage, the harmonic number and the synchronous phase of the RF cavity. Therefore by measuring $\nu_{s}$ it is possible to determine $\alpha_{1}$.

The peak RF cavity voltage, $V_{o}$, was adjusted to $350 \mathrm{kV}$. Then we measured $\nu_{s}^{2}$ as we increased the QF excitation current, $I_{Q F}$. The results are plotted in figure 4 . 


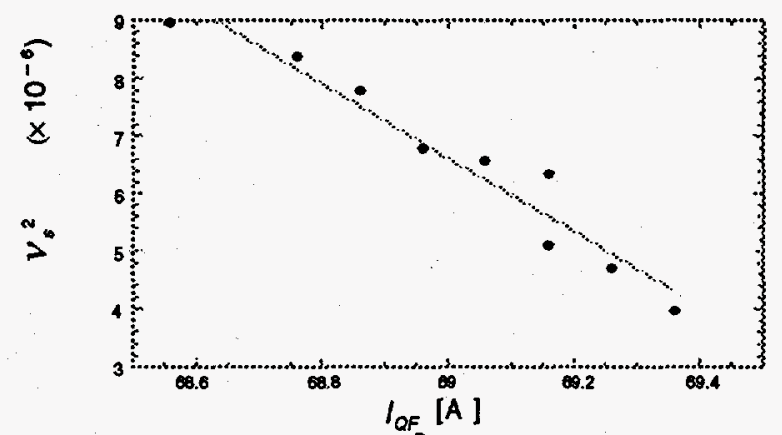

FIG. 4. Measured values of the $\nu_{s}^{2}$ verses $\mathrm{QF}$ excitation current.

The plot is linear as expected from equations 3 and 4 . From equation 4 it is possible to determine the values of $\alpha_{1}$ as a function of $I_{Q F}$. The results are plotted in figure 5. A comparison between figures 3 and 5 shows good agreement between the model and the machine.

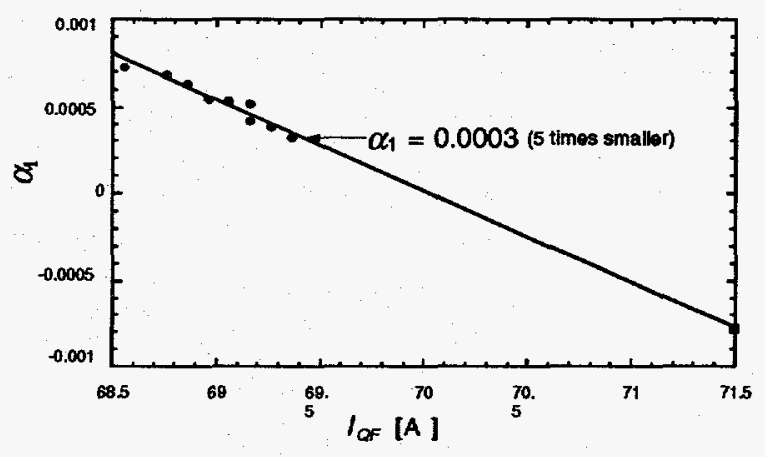

FIG. 5. The momentum compaction as a function of QF excitation current.

Horizontal Beam Displacement. A second method of measuring $\alpha_{1}$ is to measure the horizontal beam displacement as a function of RF frequency. By measuring the horizontal beam displacement, $\triangle x_{B P M}$, at a beam position monitor (BPM) resulting from a change in the RF frequency, $\Delta f_{R F}$, it is possible to measure the ratio, $-\eta / \alpha_{1}$,

$$
\alpha_{1}=-\frac{\eta_{B P M}}{f_{R F}} \frac{1}{\Delta x_{B P M} / \Delta f_{R F}}
$$

where $\eta_{B P M}$ is the horizontal dispersion at the BPM. In figure 6 we plot $\Delta x / \Delta f_{R F}$ verses BPM number for two setting of $I_{Q F}$. The shape of the curves looks like a mirror image of the dispersion function as viewed in figure $2(\mathrm{~b})$. From equation 5 we know that $\Delta x / \Delta f_{R F}$ is proportional to $-\eta / \alpha_{1}$ so if $\alpha_{1}$ is positive then $\Delta x / \Delta f_{R F}$ will be the mirror image of $\eta$. Therefore from the shape of the plot we know that $\alpha_{1}$ is positive. 


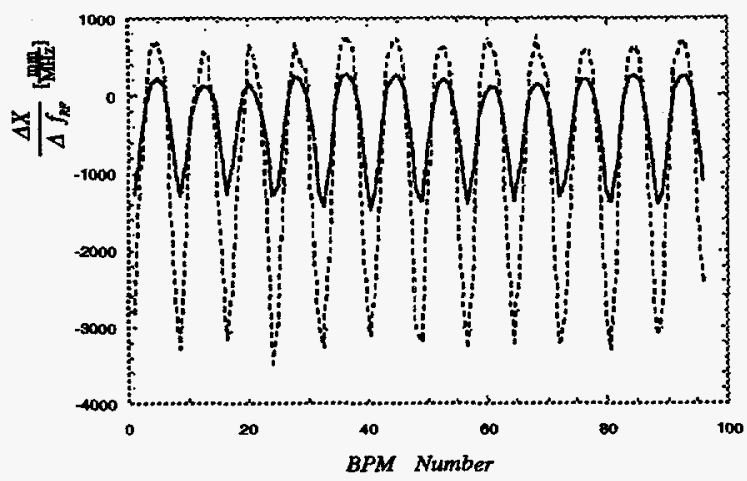

FIG. 6. Horizontal beam displacement as a function of a change in RF frequency at each beam pasition monitor.

In order to determine the magnitude of $\alpha_{1}$ from equation 5 it is necessary to assume some value for the dispersion at the BPMs. From our model of the machine we computed the dispersion in the outer straight section BPMs , BPM1 and BPM8 in figure 1 , to be $0.7 \mathrm{~m}$. Then from equation 5 we determined that $\alpha_{1}=0.001$ (solid line in figure 6) and $\alpha_{1}=0.0003$ (dashed line figure 6).

\section{Measurement of the Bunch Length}

At low currents the bunch length, $\sigma_{b}$, is:

$$
\sigma_{b}=\frac{\alpha_{1} R_{o}}{\nu_{s}} \frac{\sigma_{e}}{E_{o}}
$$

where $R_{o}$ is the ring's average radius of curvature and $\sigma_{e} / E_{o}$ is the relative energy spread of the bunch. Since $\nu_{s}$ is proportional to the square root of $\alpha_{1}$ (equation 4), $\sigma_{b}$ is proportional to the square root of $\alpha_{1}$.

The peak RF voltage, $V_{o}$, was adjusted to $1 \mathrm{MV}$. Using a streak camera with 500 femtosecond resolution we measured $\sigma_{b}$ as a function of $\nu_{s}$. The results are plotted in figure 7. In the normal lattice configuration the bunch length is $16 \mathrm{ps}$ and $\nu_{s}$ is 0.0075 . From equations 4 and 6 we expect $\sigma_{b}$ to decrease linearly with $\nu_{s}$ (solid line in figure 7). With the low alpha lattice, $\nu_{s}$ was adjusted between 0.0033 and 0.0068 . However at values of $\nu_{s}$ below 0.004 we were not able to store more than $0.2 \mathrm{~mA}$ of current in a single bunch and as a result we were not able to measure $\sigma_{b}$. The smallest value of $\nu_{s}$ at that we were able to measure $\sigma_{b}$ was 0.0046 . At this value of $\nu_{s}, \sigma_{b}$ was $8.1 \mathrm{ps}$.

At small values of $\alpha_{1}$ we observed large bunch size oscillations. In addition we occasionally saw what appeared to be a small satellite bunch orbiting the main bunch (figure 8). 


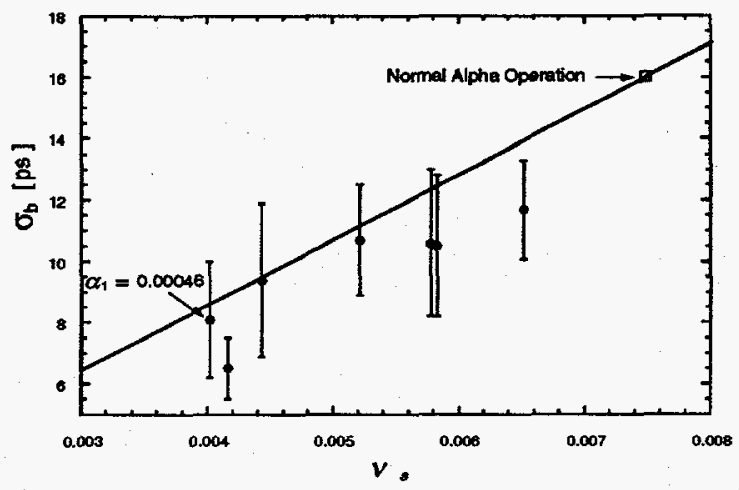

FIG. 7. Measurements of the bunchlength verses synchrotron tune.

We also saw that the bunch length was oscillating by \pm 2 ps. In addition we sometimes observed the bunch breaking into two.
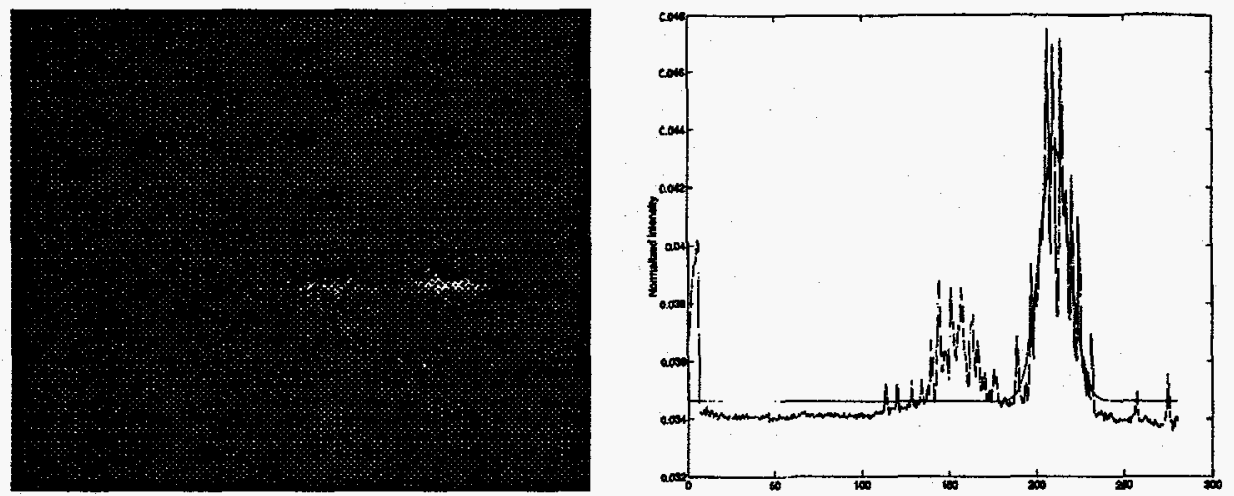

FIG. 8. Observations of a bunch satellite at small $\alpha_{1}$ with a streak camera. In the two figures the bunch is traveling from right to left. Left: horizontal size verses bunch length. Right: Intensity verses bunch length.

\section{Limits to Further Reduction of $\alpha_{1}$}

As discussed in the paper by Robin et al (3) the size of the next order momentum compaction factor $\alpha_{2}$ can limit stability of motion at small $\alpha_{1}$. For this reason we proceeded to measure $\alpha_{2}$.

If $\alpha_{2}$ is nonzero, then a change in $f_{R F}$ will result in a change in $\nu_{s}(2)$ :

$$
\nu_{s}=\nu_{s o}\left(1-4 \frac{\alpha_{2}}{\alpha_{1}^{2}} \frac{\Delta f_{R F}}{f_{R F}}\right)^{1 / 4}
$$

where $\nu_{s o}$ is the value of the synchrotron tune at $\Delta f_{R F}=0$. Figure 9 shows the measurement of $\nu_{s}$ verses $f_{R F}$. From the data and equation 7 we determined $\alpha_{2}$ to be 0.03 . The energy acceptance in the low $\alpha_{1}$ regime is $\alpha_{1} / \alpha_{2}(9)$. Therefore at our smallest measured $\alpha_{1}$ value of 0.0003 , the energy acceptance is only $1 \%$ (figure 10). For this small energy aperture we expect the Touschek effect (10) severly limits the single bunch current. Because the stable longitudinal phase space area is proportional to $\alpha_{1}$, at smaller values of $\alpha_{1}$ the stable area is too small to store 
beam. At smaller values of $\alpha_{1}$ the stable longitudinal phase space shrinks further and prevents us from storing any current.

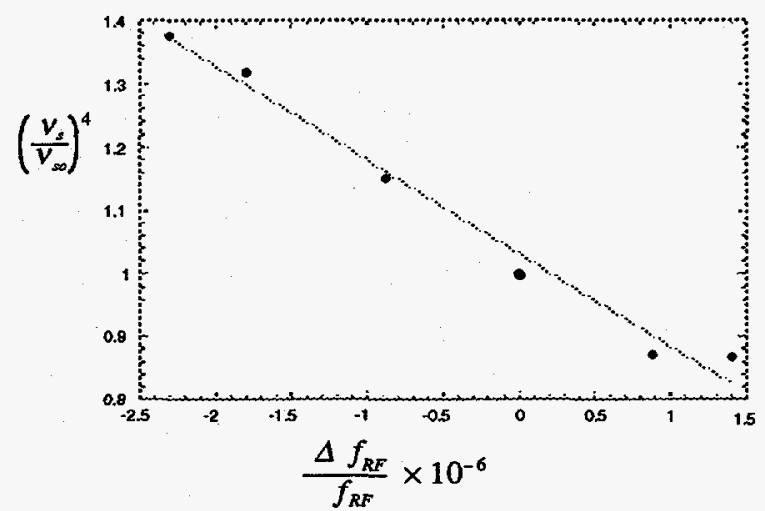

FIG. 9. Measurement of the second order momentum compaction factor.

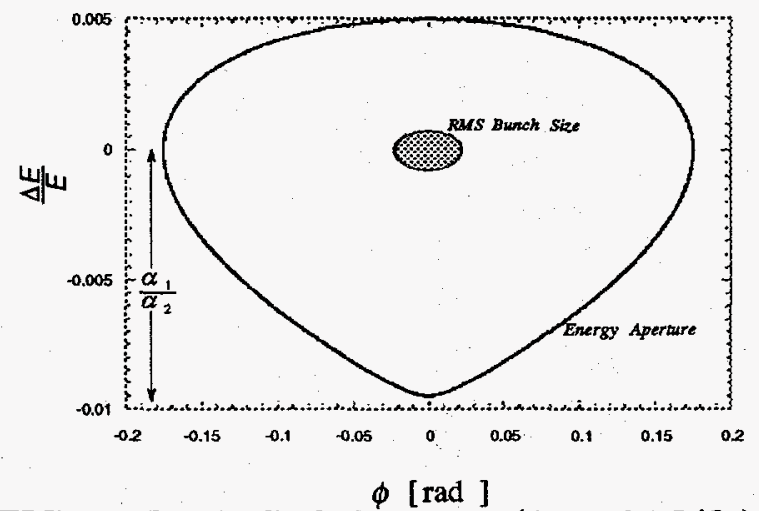

FIG. 10. Longitudinal phase space $\left(\phi=2 \pi h \Delta L / L_{0}\right)$

In principle we can reduce $\alpha_{2}$ by changing sextupole fields in regions of large dispersion (3). Unfortunately, in the present low alpha lattice, both sextupole families are located in regions of small dispersion (table 1). Therefore we are unable to change $\alpha_{2}$.

\section{NEGATIVE MOMENTUM COMPACTION}

Higher peak currents and shorter bunch lengthening have been predicted by Fang et al. (8) and measured by Nadji et al at Super-ACO $(5,3)$ when operating with a negative momentum compaction factor. Based upon the success of these experiments, we have have begun to explore negative $\alpha_{1}$ operation of the ALS.

In order to adjust $I_{Q F}$ to make $\alpha_{1}$ negative, we fit the data displayed in figure 4 with a straight line (solid line in figure 4) and then extrapolate to negative $\alpha_{1}$. For a value of $I_{Q F}=71.5 \mathrm{~A}$, we expected a value of $\alpha_{1}=-0.0008$ (square dot in figure 4).

$I_{Q F}$ was then adjusted to $71.5 \mathrm{~A}$ and the phase of the RF cavity, $\phi_{o}$, was shifted by $180 \mathrm{deg}$ with respect to the injector. The peak RF cavity voltage was set to 1 MV. Under these conditions we were able to store beam current - about $1 \mathrm{~mA}$ in a single bunch. 


\section{Characterizing the Negative $\alpha_{1}$ Lattice}

Because we injected and stored beam with the RF phase shifted by $180 \mathrm{deg}$ we had confidence that $\alpha_{1}$ was negative. But to definitively check the sign of $\alpha_{1}$ we measured the horizontal beam displacement as a function of the RF cavity frequency. The results are plotted as dashed lines in figure 11. We have also plotted as a solid line in figure 11 our measured data for positive $\alpha_{1}$ (same as the solid line in figure 6)

As can be seen in figure 11 the dashed line is "flipped" with respect to the solid line. This is expected from equation 5 and it clearly says that $\alpha_{1}$ is negative.

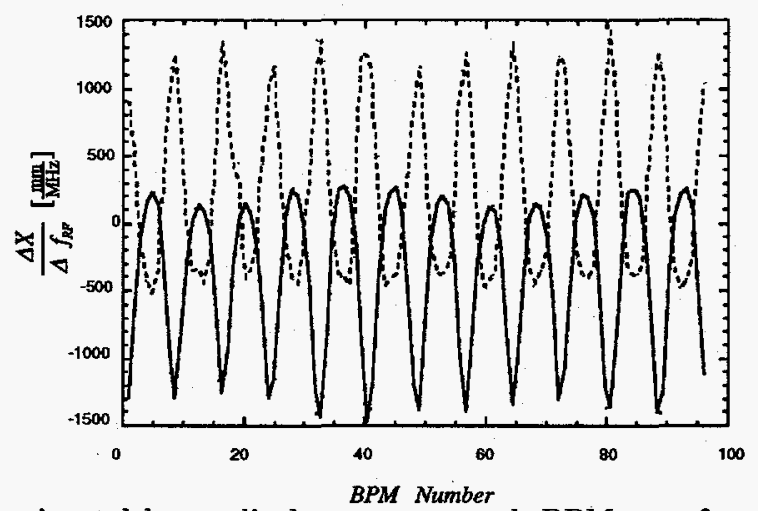

FIG. 11. Measured horizontal beam displacement at each BPM as a function of RF cavity frequency. Solid line : $\alpha_{1}=0.001$. Dashed line : $\alpha_{1}=-0.0011$.

Streak camera images show that at negative $\alpha_{1}$ the beam distribution is leaning backwards in time (figure 12). At positive $\alpha_{1}$ distribution is leaning forwards in time. This is consistent with results by Fang et al (8) who have simulated the effects of potential-well distortion. In addition the beam was noticeably more stable at negative $\alpha_{1}$.

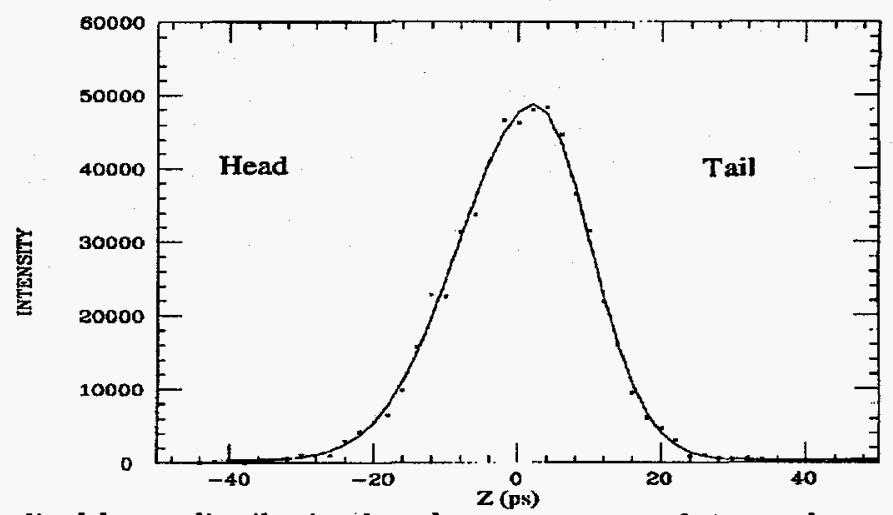

FIG. 12. Longitudinal beam distribution based on an average of 17 streak camera images taken with $0.5 \mathrm{~mA}$ in a single bunch and $\alpha_{1}=-0.0011$.

\section{SUMMARY OF RESULTS}

By just changing quadrupole strengths we have detuned the ALS lattice in order to reduce $\alpha_{1}$. At this time we have been able to reduce $\alpha_{1}$ by a factor of 5 to 0.0003 
and the bunch length by a factor of 2 to 8 ps. However at 8 ps we were only able to store $0.1 \mathrm{~mA}$ of current in a single bunch. The size of $\alpha_{2}$ was measured to be 0.03 and it was determined to be the limit to further reduction of $\alpha_{1}$. In addition we also stored current in a negative $\alpha_{1}$ lattice.

By imaging the beam with a streak camera, we have seen evidence of the on-set of the head-tail instability and satellite bunches in the positive $\alpha_{1}$ configuration. We have also observed a shifts in beam tail distributions when operating with a positive and negative $\alpha_{1}$.

In the future we will attempt to correct the $\alpha_{2}$ in order to reduce $\alpha_{1}$ further. Also we will further investigate negative $\alpha_{1}$ operation.

\section{Acknowledgements}

We with to thank Robert Siemann, Mateas Hirsch, Hideaki Ohgaki, John Byrd and Albert Hofmann for many interesting discussions about the experiment and results. We with to thank the Stanford Linear Accelerator Center for allowing us to use their streak camera to measure bunch lengths. Finally we wish to acknowledge the contributions from the ALS staff.

\section{REFERENCES}

1. H. Hama, S. Takano, and B. Isoyoma, Nucl. Instr. and Meth. in Phys. Res. A329 (1993) 29-36.

2. H. Hama et al. "Operation with the Low Momentum Compaction Factor on an Electron Storage Ring", Proceedings of the 9th Symposium on Accelerator Science and Technology, Tsukuba, Japan (1993) 468.

3. D. Robin, H. Hama, and A. Nadji, "Experimental Results in Low Alpha Rings", in this workshop's proceedings this workshop (1995).

4. A. Nadji et al, "Quasi-Isochronous Optics for Super-ACO", Proceedings of the 4th European Particle Accelerator Conference, (1994) 128.

5. A. Nadji et al, "Experiments with the Super-ACO storage ring operating in a quasi-isochronous mode", to be published.

6. M. Sands, "The Physics of Electron Storage rings: an Introduction", Stanford Linear Accelerator Note, SLAC-121, (1970)

7. $\alpha_{1}$ was calculated verses quadrupole gradient, $k_{Q F}$, using MAD and the gradients were transformed to excitation current. For a MAD reference see $H$. Grote and F. Iselin, CERN report CERN/SL/90-13 (1991).

8. S.X. Fang et al, KEK Preprint 94-190 (1995).

9. D. Robin et al, Phys. Rev. E 48,3 (1993) 2153.

10. J. LeDuff, Nucl. Instr. and Meth. A239 (1985) 83 .

\section{DISCLAIMER}

This report was prepared as an account of work sponsored by an agency of the United States Government. Neither the United States Government nor any agency thereof, nor any of their employees, makes any warranty, express or implied, or assumes any legal liability or responsibility for the accuracy, completeness, or usefulness of any information, apparatus, product, or process disclosed, or represents that its use would not infringe privately owned rights. Reference herein to any specific commercial product, process, or service by trade name, trademark, manufacturer, or otherwise does not necessarily constitute or imply its endorsement, recommendation, or favoring by the United States Government or any agency thereof. The views and opinions of authors expressed herein do not necessarily state or reflect those of the United States Government or any agency thereof. 